\title{
Cholesterol goal achievement and lipid-lowering therapy in patients with stable or acute coronary heart disease in Singapore: results from the Dyslipidemia International Study II
}

Kian-Keong Poh ${ }^{1,2}$, FRCP, FACC, Chee Tang Chin ${ }^{3,4}$, MBChB, Khim Leng Tong ${ }^{5}$, MBBS, MRCP, Julian Ko Beng Tan ${ }^{6}$, FRCP,

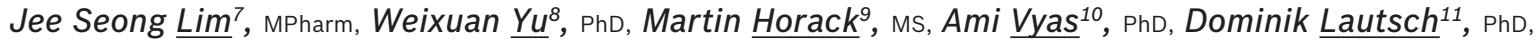
Baishali Ambegaonkar ${ }^{11}$, PhD, Philippe Brudi ${ }^{11}$, MD, Anselm K Gitt $^{9,12}$, MD

INTRODUCTION Dyslipidaemia is a major risk factor for coronary heart disease (CHD). There is a lack of data on the extent of lipid abnormalities and lipid-lowering therapy (LLT) in Singapore.

METHODS The Dyslipidemia International Study (DYSIS) II was a multinational observational study of patients with stable CHD and hospitalised patients with an acute coronary syndrome (ACS). A full lipid profile and use of LLT were documented at baseline, and for the ACS cohort, at four months post-hospitalisation.

RESULTS 325 patients were recruited from four sites in Singapore; 199 had stable CHD and 126 were hospitalised with an ACS. At baseline, $96.5 \%$ of the CHD cohort and $66.4 \%$ of the ACS cohort were being treated with LLT. In both cohorts, low-density lipoprotein cholesterol (LDL-C) levels were lower for the treated than the non-treated patients; accordingly, a higher proportion of patients met the LDL-C goal of $<70 \mathrm{mg} / \mathrm{dL}$ (CHD: $28.1 \%$ vs. $0 \%, p=0.10$; ACS: $20.2 \%$ vs. $0 \%$, $p<0.01$ ). By the four-month follow-up, a higher proportion of the ACS patients that were originally not treated with LLT had met the LDL-C goal (from $0 \%$ to $54.5 \%$ ), correlating with the increased use of medication. However, there was negligible improvement in the patients who were treated prior to the ACS.

CONCLUSION Dyslipidaemia is a significant concern in Singapore, with few patients with stable or acute CHD meeting the recommended European Society of Cardiology/European Atherosclerosis Society goal. LLT was widely used but not optimised, indicating considerable scope for improved management of these very-high-risk patients.

Keywords: acute coronary syndrome, coronary artery disease, ezetimibe, hyperlipidaemia, statins

\section{INTRODUCTION}

Coronary heart disease (CHD) is the largest contributor to cardiovascular death across the world. ${ }^{(1)}$ With approximately half of the global burden of cardiovascular disease being located in Asia, rates of mortality due to $\mathrm{CHD}$ are alarmingly high in this region. ${ }^{(2)}$ Singapore is one of the most developed countries in Southeast Asia, and displays similar cardiovascular mortality rates to those of European and North American countries. ${ }^{(2,3)}$ With rising levels of obesity and Type 2 diabetes mellitus (DM), the situation is set to get worse over the coming years. ${ }^{(4)}$ Effective management of the risk factors associated with CHD is therefore essential for reducing the burden of cardiovascular disease in Singapore.

Dyslipidaemia is a prominent risk factor, with one Asian study reporting that for every $1 \mathrm{mmol} / \mathrm{L}(39 \mathrm{mg} / \mathrm{dL}$ ) increase in total cholesterol (TC), there is an approximately $35 \%$ increased risk of coronary death. ${ }^{(5)}$ It was previously shown that a reduction in low-density lipoprotein cholesterol (LDL-C) of $1 \mathrm{mmol} / \mathrm{L}$ $(39 \mathrm{mg} / \mathrm{dL})$ can reduce the five-year risk of major vascular events by approximately one-fifth; ${ }^{(6)}$ therefore, guidelines on the management of lipid abnormalities generally focus on the lowering of this particular lipid. (7-9) The European Society of Cardiology (ESC) and the European Atherosclerosis Society (EAS) advocate an LDL-C goal of $<70 \mathrm{mg} / \mathrm{dL}$ for patients deemed to be at very high risk, ${ }^{(7)}$ while the Ministry of Health, Singapore, recommends a level of $<80 \mathrm{mg} / \mathrm{dL} .{ }^{(10)}$ On the other hand, the American College of Cardiology (ACC) and American Heart Association (AHA) guidelines do not advocate lowering LDL-C to a specific level but state that high-intensity statin therapy should be initiated or continued for all patients aged 75 years or younger with established atherosclerotic cardiovascular disease. ${ }^{(8)}$

A variety of drug classes can be used to lower lipid levels, the most commonly prescribed being statins. However, despite widespread use of lipid-lowering therapy (LLT), many patients fail to meet the ESC's recommended LDL-C goals. In the global Dyslipidemia International Study (DYSIS), only $26.8 \%$ of the 57,885 statin-treated subjects attained their risk-based LDL-C goal, including $21.7 \%$ of patients who were at very high risk..$^{(11)}$ In the Centralized Pan-Asian Survey on the Undertreatment of Hypercholesterolemia, goal achievement for very-high-risk LLTtreated hypercholesterolaemic patients was higher, at $34.9 \%$, but

\footnotetext{
'Department of Cardiology, National University Heart Centre Singapore, National University Health System, ${ }^{2}$ Yong Loo Lin School of Medicine, National University of Singapore, ${ }^{3}$ Department of Cardiology, National Heart Centre Singapore, ${ }^{4}$ Duke-NUS Medical School, ${ }^{5}$ Department of Cardiology, Changi General Hospital, ${ }^{6}$ Mount Elizabeth Medical Centre, Singapore, ${ }^{7}$ Merck Sharp \& Dohme (Malaysia) Sdn Bhd, Petaling Jaya, Selangor, Malaysia, ${ }^{8}$ MSD Pharma (Singapore) Pte Ltd, Singapore, ${ }^{9}$ Herzzentrum Ludwigshafen, Cardiology, Stiftung Institut für Herzinfarktforschung, Ludwigshafen, Germany, ${ }^{10}$ University of Rhode Island, College of Pharmacy, Department of Pharmacy Practice, Kingston, RI, ${ }^{11}$ Merck \& Co Inc, Kenilworth, NJ, USA, ${ }^{12}$ Klinikum der Stadt Ludwigshafen, Medizinische Klinik B, Ludwigshafen, Germany

Correspondence: A/Prof Kian-Keong Poh, Department of Cardiology, National University Heart Centre, National University Health System, 1E Kent Ridge Road, NUHS Tower Block Level 9, Singapore 119228. kian_keong_poh@nuhs.edu.sg
} 
still greatly inadequate. ${ }^{(12)}$ In Singapore, in a single-centre study involving patients that had suffered an acute coronary syndrome (ACS), $36.7 \%$ of patients had LDL-C goal achievement at four months after the event; however, this declined to $23.4 \%$ at the 12-month follow-up. ${ }^{(13)}$ The low proportion of patients meeting goals suggests that there is much room for improvement in the use of LLT. However, there is little available country-specific information concerning current practices.

DYSIS II is a multinational observational study that was established to quantify the extent of hyperlipidaemia and its treatment, both globally and in individual countries. ${ }^{(14,15)}$ Using standardised methodology, data was collected from patients with either chronic or acute CHD in order to evaluate their lipid levels and use of LLT. This study presents the data collected from patients in Singapore.

\section{METHODS}

DYSIS II was an observational, cross-sectional, multinational study. Patients in Singapore were enrolled at four sites from January 2014 to August 2014. Patients over 18 years of age were included if they were either attending an outpatient physician visit for stable CHD or were being hospitalised with an ACS. Patients were excluded if they were participating in a clinical trial at the same time as the study and, for the ACS cohort, if they did not survive until hospital discharge.

Stable CHD was defined as stenosis > 50\% (as shown by coronary angiography or cardiac computed tomography), prior percutaneous coronary intervention $(\mathrm{PCl})$ or prior coronary artery bypass grafting. ACS was defined as an ST segment elevation myocardial infarction or left bundle branch block myocardial infarction (STEMI/LBBB-MI), a non-ST segment elevation myocardial infarction or unstable angina. An available full lipid profile was required for all patients. For the $\mathrm{CHD}$ cohort, this was constructed from the last blood test taken within the previous 12 months. For the ACS cohort, this was constructed from blood taken within 24 hours of hospital admission. Patients were categorised according to whether or not they were being treated with LLT at baseline. In order to be included in the LLT groups, treatment duration had to be at least three months by the time of the lipid test. Data on the CHD patients was collected at the physician visit, while data on the ACS patients was collected at hospital admission and during a telephone interview four months later. The study received approval from the ethics committee at each site and was conducted in accordance with the Declaration of Helsinki and its amendments. All patients provided written informed consent.

Data was entered into an online database maintained at the Institut für Herzinfarktforschung in Ludwigshafen, Germany. For all patients, demographic and clinical variables were documented at baseline. These included age, gender, ethnicity and body mass index (BMI); the presence of hypertension, DM, chronic kidney disease (CKD) or congestive heart failure (CHF); documentation of prior stroke or myocardial infarction; and the presence of other cardiovascular risk factors, including smoking, a sedentary lifestyle and a family history of CHD. Obesity was defined as $\mathrm{BMI}>30 \mathrm{~kg} / \mathrm{m}^{2}$. Hypertension was defined as the presence of current treatment to lower blood pressure, a previous diagnosis of hypertension, or blood pressure level > 140/90 mmHg. DM was defined as the presence of current treatment for DM, a previous diagnosis of DM or fasting plasma glucose level $\geq 126 \mathrm{mg} / \mathrm{dL}$. A sedentary lifestyle was defined as $<20-30$ minutes of walking for $<3-4$ days per week.

The baseline lipid profile included serum levels of LDL-C, high-density lipoprotein cholesterol (HDL-C), non-HDL-C, TC and triglycerides. Each ACS patient was assigned a preadmission cardiovascular risk status as defined in the $2011 \mathrm{ESC} /$ EAS guidelines, which depended on the risk factors that they displayed prior to the index ACS event. ${ }^{(16)}$ Definitions of very high risk and high risk included the presence of comorbidities such as DM and CKD, while those for moderate and low risk took into account additional risk factors or markers, such as obesity or high C-reactive protein (CRP). Each risk level was associated with an LDL-C goal $(<70 \mathrm{mg} / \mathrm{dL},<100 \mathrm{mg} / \mathrm{dL},<115 \mathrm{mg} / \mathrm{dL}$ and $<130 \mathrm{mg} / \mathrm{dL}$ for very-high-risk, high-risk, moderate risk and low-risk patients, respectively), and the proportions of patients who met their goal were calculated. LDL-C goal achievement was evaluated again at the four-month follow-up, during which all patients were considered to be at very high risk due to their ACS event. Hypothetical changes in goal achievement, based on treatment intensification for LLT-treated patients who were not at their goal, were calculated based on the reported effects of the different statins and ezetimibe in lowering LDL-C. ${ }^{(17,18)}$ These changes included doubling the statin dose for any patients who had not attained their goal and were not being treated with atorvastatin $80 \mathrm{mg}$. An additional LDL-C reduction of $24 \%$ could be expected from adding ezetimibe to the treatment regimen.

The specific LLT treatment that patients received was recorded at baseline and, for the ACS cohort, at the four-month follow-up interview. The statins investigated were atorvastatin, fluvastatin, lovastatin, pitavastatin, pravastatin, rosuvastatin and simvastatin. Non-statins included ezetimibe, nicotinic acid, fibrates and omega-3 fatty acids. Both monotherapy and combination therapies were detailed. Statin dosages were normalised to atorvastatin potency according to clinical trial data on the lipidlowering abilities of the different statins. ${ }^{(18)}$ For the ACS cohort, the occurrence of adverse events since hospital discharge was documented at the four-month follow-up. These included death, rehospitalisation and non-fatal events.

SAS version 9.3 (SAS Institute Inc, Cary, NC, USA) was used for the statistical analyses. Continuous variables were presented as mean \pm standard deviation, or median and interquartile range. Categorical variables were presented as absolute values and percentages. Differences between the LLT and no-LLT groups of each cohort were analysed using chi-square or Mann-Whitney-Wilcoxon test. Predictors of meeting the LDL-C goal of $<70 \mathrm{mg} / \mathrm{dL}$ among LLT-treated patients were assessed using multivariate regression analysis, including the following covariates: age, gender, BMI, current smoking, sedentary lifestyle, waist circumference, hypertension, Type 2 DM, stable angina, $\mathrm{CKD}$, history of $\mathrm{CHF}$ and statin dose. Mortality at follow-up was estimated using Kaplan-Meier analysis, with p-values calculated 
Table I. Characteristics of patients who attended an outpatient appointment for stable coronary heart disease (CHD).

\begin{tabular}{|c|c|c|c|c|}
\hline \multirow[t]{2}{*}{ Parameter } & \multicolumn{3}{|c|}{ No. $(\%) /$ mean \pm SD } & \multirow[t]{2}{*}{ p-value* } \\
\hline & All patients $(n=199)$ & LLT $(n=192)$ & No LLT $(n=7)$ & \\
\hline Age (yr) & $64.8 \pm 9.6$ & $64.6 \pm 9.7$ & $70.4 \pm 5.7$ & 0.07 \\
\hline Male gender & $157(78.9)$ & $152(79.2)$ & $5(71.4)$ & 0.62 \\
\hline \multicolumn{5}{|l|}{ Ethnicity } \\
\hline Chinese & $88(44.2)$ & $82(42.7)$ & $6(85.7)$ & $<0.05$ \\
\hline Indian & $15(7.5)$ & $15(7.8)$ & $0(0)$ & 0.44 \\
\hline Filipino & $1(0.5)$ & $0(0)$ & $0(0)$ & 0.85 \\
\hline Other Asian & $87(43.7)$ & $86(44.8)$ & $1(14.3)$ & 0.11 \\
\hline Other & $8(4.0)$ & $8(4.2)$ & $0(0)$ & 0.58 \\
\hline BMI $\left(\mathbf{k g} / \mathbf{m}^{2}\right)$ & $26.4 \pm 4.2$ & $26.4 \pm 4.2$ & $26.4 \pm 4.2$ & 0.17 \\
\hline $\mathrm{BMI}>30 \mathrm{~kg} / \mathrm{m}^{2}$ & $34(17.1)$ & $33(17.2)$ & $1(14.3)$ & 0.84 \\
\hline \multicolumn{5}{|l|}{ Comorbidities/CV risk factors } \\
\hline Type 2 diabetes mellitus & 77/198 (38.9) & 73/191 (38.2) & $4(57.1)$ & 0.31 \\
\hline Hypertension & $144(72.4)$ & $140(72.9)$ & $4(57.1)$ & 0.36 \\
\hline CKD & $13(6.5)$ & $12(6.3)$ & $1(14.3)$ & 0.40 \\
\hline History of stroke ${ }^{\dagger}$ & $13 / 195(6.7)$ & 13/188 (6.9) & $0(0)$ & 0.47 \\
\hline PAD & $2 / 195(1.0)$ & $2 / 188(1.1)$ & $0(0)$ & 0.78 \\
\hline Current smoker & $26(13.1)$ & $25(13.0)$ & $1(14.3)$ & 0.92 \\
\hline Sedentary lifestyle & $41 / 198(20.7)$ & 37/191 (19.4) & $4(57.1)$ & $<0.05$ \\
\hline Family history of $\mathrm{CHD}$ & $72 / 193(37.3)$ & $72 / 186(38.7)$ & $0(0)$ & $<0.05$ \\
\hline \multicolumn{5}{|l|}{ CHD diagnosis } \\
\hline Stenosis > 50\% (CA) & $77(38.7)$ & 75 (39.1) & $2(28.6)$ & 0.58 \\
\hline Stenosis $>50 \%$ (cardiac CT) & $2(1.0)$ & $2(1.0)$ & $0(0)$ & 0.79 \\
\hline Prior $\mathrm{PCl}$ & $125(62.8)$ & $122(63.5)$ & $3(42.9)$ & 0.27 \\
\hline Prior CABG & $46(23.1)$ & $45(23.4)$ & $1(14.3)$ & 0.57 \\
\hline History of $\mathrm{ACS}^{\ddagger}$ & $144(72.4)$ & $138(71.9)$ & $6(85.7)$ & 0.42 \\
\hline
\end{tabular}

*LLT vs. no LLT, calculated using chi-square test or Mann-Whitney-Wilcoxon test. †ncluding ischaemic and haemorrhagic stroke. $¥>3$ mth prior to enrolment. ACS: acute coronary syndrome; BMI: body mass index; CA: coronary angiography; CABG: coronary artery bypass grafting; CKD: chronic kidney disease; CT: computed tomography; CV: cardiovascular; LLT: lipid-lowering therapy; PAD: peripheral artery disease; PCl: percutaneous coronary intervention; SD: standard deviation

using a log-rank test. A p-value $<0.05$ was considered statistically significant for all comparisons.

\section{RESULTS}

A total of 325 patients were enrolled in the Singapore cohort of DYSIS II. Of these, 199 patients were attending an outpatient appointment for stable CHD and 126 were being admitted to hospital after an ACS.

The mean age of the stable CHD cohort was 64.8 years and $78.9 \%$ were male (Table I). The majority of these patients $(96.5 \%)$ were being treated with LLT, and had no significant differences with the group that did not receive LLT in terms of age, gender or $\mathrm{BMI}$. Comorbidities and cardiovascular risk factors were extremely common, particularly hypertension (72.4\%), Type 2 DM (38.9\%), a family history of CHD (37.3\%) and a sedentary lifestyle (20.7\%). There were few significant differences in rates of comorbidities and risk factors between the two groups. In the patients not being treated with LLT, a sedentary lifestyle was more common, while a family history of CHD was less common. Patients of different ethnicities had similar characteristics, although obesity (Chinese 11.4\%, Indian 26.7\%, other Asian 18.2\%; p < 0.05) and Type 2 DM (Chinese $43.2 \%$, Indian $66.7 \%$, other Asian $31.0 \%$; p < 0.05 ) were more prevalent in Indian patients, and a higher proportion of Chinese patients reported having a sedentary lifestyle (Chinese 29.5\%, Indian 6.7\%, other Asian 11.5\%; p < 0.01).

The ACS cohort had a mean age of 61.0 years and $81.7 \%$ were male (Table II). Patients who were being treated with LLT were significantly older than those not being treated with LLT (63.3 years vs. 56.5 years; $p<0.01$ ). A number of comorbidities were more common in the LLT group than the no-LLT group, including hypertension ( $88.1 \%$ vs. $50.0 \%$; p $<0.0001)$, Type 2 DM $(57.1 \%$ vs. $33.3 \%$; $p<0.05)$ and CKD $(25.0 \%$ vs. $9.5 \% ; \mathrm{p}<0.05)$. On the other hand, a smaller proportion of the LLT patients were current smokers $(21.4 \%$ vs. $45.2 \%$; $p<0.01)$. A diagnosis of STEMI/LBBB-MI was less common for the LLT group than the no-LLT group $(23.8 \%$ vs. $45.2 \%$; $p<0.05)$, while unstable angina was slightly more common $(27.4 \%$ vs. $11.9 \% ; \mathrm{p}<0.05)$. The only notable difference among patients of different ethnicities was the higher prevalence of a sedentary lifestyle in the Chinese group (Chinese 39.6\%, Indian 18.2\%, other Asian 9.1\%; $p<0.01$ ).

The mean LDL-C level of the CHD cohort was $86.2 \pm$ $28.1 \mathrm{mg} / \mathrm{dL}$ (i.e. $2.23 \pm 0.73 \mathrm{mmol} / \mathrm{L}$ ), with the LLT-treated patients having a significantly lower level than the no-LLT 
Table II. Characteristics of patients who were admitted for ACS.

\begin{tabular}{|c|c|c|c|c|}
\hline \multirow[t]{2}{*}{ Parameter } & \multicolumn{3}{|c|}{ No. $(\%) /$ mean \pm SD } & \multirow[t]{2}{*}{ p-value* } \\
\hline & All patients $(n=126)$ & LLT $(n=84)$ & No LLT $(n=42)$ & \\
\hline Age (yr) & $61.0 \pm 11.7$ & $63.3 \pm 11.7$ & $56.5 \pm 10.3$ & $<0.01$ \\
\hline Male gender & $103(81.7)$ & $61(72.6)$ & $42(100.0)$ & $<0.001$ \\
\hline \multicolumn{5}{|l|}{ Ethnicity } \\
\hline Chinese & $49(38.9)$ & $32(38.1)$ & $17(40.5)$ & 0.80 \\
\hline Indian & $11(8.7)$ & $7(8.3)$ & $4(9.5)$ & 0.82 \\
\hline Other Asian & $55(43.7)$ & $39(46.4)$ & $16(38.1)$ & 0.37 \\
\hline Other & $11(8.7)$ & $6(7.1)$ & $5(11.9)$ & 0.37 \\
\hline BMI $\left(\mathbf{k g} / \mathbf{m}^{2}\right)$ & $25.9 \pm 4.4$ & $26.1 \pm 4.3$ & $25.4 \pm 4.6$ & 0.27 \\
\hline $\mathrm{BMI}>30 \mathrm{~kg} / \mathrm{m}^{2}$ & $23(18.3)$ & $18(21.4)$ & $5(11.9)$ & 0.19 \\
\hline \multicolumn{5}{|l|}{ Comorbidities/CV risk factors } \\
\hline Type 2 diabetes mellitus & $62(49.2)$ & $48(57.1)$ & $14(33.3)$ & $<0.05$ \\
\hline Hypertension & $95(75.4)$ & $74(88.1)$ & $21(50.0)$ & $<0.0001$ \\
\hline CKD & $25(19.8)$ & $21(25.0)$ & $4(9.5)$ & $<0.05$ \\
\hline History of stroke ${ }^{\dagger}$ & 9/123 (7.3) & $7 / 82(8.5)$ & $2 / 41(4.9)$ & 0.46 \\
\hline PAD & $2(1.6)$ & $2(2.4)$ & $0(0)$ & 0.31 \\
\hline Current cigarette smoker & $37(29.4)$ & $18(21.4)$ & $19(45.2)$ & $<0.01$ \\
\hline Sedentary lifestyle & $29 / 125(23.2)$ & $21(25.0)$ & $8 / 41(19.5)$ & 0.49 \\
\hline Family history of CHD & $52 / 122(42.6)$ & $36(42.9)$ & $16 / 38(42.1)$ & 0.94 \\
\hline \multicolumn{5}{|l|}{ ACS diagnosis } \\
\hline STEMI/LBBB-MI & $39(31.0)$ & $20(23.8)$ & $19(45.2)$ & $<0.05$ \\
\hline NSTEMI & $59(46.8)$ & $41(48.8)$ & $18(42.9)$ & 0.53 \\
\hline Unstable angina & $28(22.2)$ & $23(27.4)$ & $5(11.9)$ & $<0.05$ \\
\hline
\end{tabular}

Value of $\mathrm{n}$ is stated when missing data was excluded from the analysis. *LLT vs. no LLT, calculated using chi-square test or Mann-Whitney-Wilcoxon test. †ncludes ischaemic and haemorrhagic stroke. ACS: acute coronary syndrome; BMI: body mass index; CV: cardiovascular; CHD: coronary heart disease; CKD: chronic kidney disease; LLT: lipid-lowering therapy; PAD: peripheral artery disease; SD: standard deviation; STEMI/LBBB-MI: ST segment elevation myocardial infarction/left bundle branch block myocardial infarction; NSTEMI: non-ST elevation myocardial infarction

patients $(84.8 \mathrm{mg} / \mathrm{dL}$ vs. $125.3 \mathrm{mg} / \mathrm{dL} ; \mathrm{p}<0.01)$ (Table III). Median HDL-C values did not vary greatly between the LLT and no-LLT groups (43.5 mg/dL vs. $45.0 \mathrm{mg} / \mathrm{dL} ; \mathrm{p}=0.86)$, nor did triglyceride levels (105.5 mg/dL vs. $115.0 \mathrm{mg} / \mathrm{dL} ; \mathrm{p}=0.82)$. Few of these very-high-risk patients had an LDL-C level below the $70 \mathrm{mg} / \mathrm{dL}$ goal $(28.1 \%$ and $0 \%$ for the LLT and no-LLT groups, respectively; $p=0.10)$. The median distance to this goal was calculated to be $19.0 \mathrm{mg} / \mathrm{dL}$ for the LLT-treated patients and $65.0 \mathrm{mg} / \mathrm{dL}$ for those who were not treated. When achievement of the Singapore guideline-recommended goal of $<80 \mathrm{mg} / \mathrm{dL}$ was evaluated, goal achievement for the overall cohort was higher $(45.7 \%)$, while $75.4 \%$ achieved the $<100 \mathrm{mg} / \mathrm{dL}$ level that was previously used in Singapore. Among the variables entered into the logistic regression model, only Type 2 DM was associated with the increased likelihood of an LLT-treated CHD patient meeting the LDL-C goal of $<70 \mathrm{mg} / \mathrm{dL}$ (OR 2.05, 95\% confidence interval 1.01-4.16; $p=0.05$ ) (Table IV).

For the ACS cohort, the mean LDL-C level at hospital admission was $113.5 \pm 43.9 \mathrm{mg} / \mathrm{dL}$ (i.e. $2.94 \pm 1.13 \mathrm{mmol} / \mathrm{L}$ ). The LLT-treated patients had a much lower LDL-C value than the noLLT patients (96.1 mg/dL vs. $148.4 \mathrm{mg} / \mathrm{dL} ; \mathrm{p}<0.0001)$ (Table III). The median triglyceride level was also lower for the LLT group (127.5 mg/dL vs. $159.0 \mathrm{mg} / \mathrm{dL}$; $\mathrm{p}<0.05)$, while HDL-C levels did not vary greatly $(41.0 \mathrm{mg} / \mathrm{dL}$ vs. $42.0 \mathrm{mg} / \mathrm{dL} ; \mathrm{p}=0.52)$. Very few patients $(13.5 \%$ ) had an LDL-C level $<70 \mathrm{mg} / \mathrm{dL}$, consisting of $17(20.2 \%)$ patients from the LLT group and no patients from the no-LLT group $(p<0.01)$. The distance to this LDL-C goal was significantly smaller for the treated patients $(33.0 \mathrm{mg} / \mathrm{dL})$ than for those not treated $(76.0 \mathrm{mg} / \mathrm{dL} ; \mathrm{p}<0.0001)$. Overall achievement of the LDL-C goal of $<80 \mathrm{mg} / \mathrm{dL}$, advocated for very-high-risk patients in the Singapore guidelines, was only slightly higher (23.8\%) than that of the ESC/EAS goal. $43.7 \%$ of the ACS cohort reached the previously used goal of $100 \mathrm{mg} / \mathrm{dL}$. The majority (87.3\%) of the ACS cohort had been deemed to be at very high cardiovascular risk even before the ACS that they were hospitalised for. For this risk category, achievement of the ESC/EAS LDL-C goal $(<70 \mathrm{mg} / \mathrm{dL})$ was low, with $21.1 \%$ of the LLT-treated patients and none of those not treated meeting this goal (Fig. 1a). Goal achievement for the small numbers of patients in the lower risk categories was slightly better but remained inadequate, especially for those not being treated with LLT. None of the variables entered into the logistic regression model were found to be associated with LLT-treated ACS patients with an LDL-C level $<70 \mathrm{mg} / \mathrm{dL}$ (Table IV).

At the four-month follow-up, 66 (61.1\%) of 108 ACS patients had a further lipid profile available (Fig. 1b). In this group, achievement of the $70 \mathrm{mg} / \mathrm{dL}$ LDL-C goal remained poor, at 34.8\% overall. For those treated with LLT prior to the index ACS, goal achievement increased only slightly, from $22.7 \%$ to $25.0 \%$. For those that were not being treated prior to the ACS, the attainment rate rose from $0 \%$ to $54.5 \%$. 
Table III. Lipid profile at baseline.

\begin{tabular}{|c|c|c|c|c|}
\hline \multirow[t]{3}{*}{ Parameter } & \multicolumn{4}{|c|}{ No. $(\%) /$ mean \pm SD/median (IQR) } \\
\hline & \multicolumn{4}{|c|}{ Stable coronary heart disease } \\
\hline & All patients & LLT & No LLT & p-value \\
\hline & $(n=199)$ & $(n=192)$ & $(n=7)$ & \\
\hline LDL-C (mg/dL) & $86.2 \pm 28.1$ & $84.8 \pm 27.0$ & $125.3 \pm 33.1$ & $<0.01$ \\
\hline$<70 \mathrm{mg} / \mathrm{dL}^{*}$ & $54(27.1)$ & $54(28.1)$ & $0(0)$ & 0.10 \\
\hline$<80 \mathrm{mg} / \mathrm{dL}^{+}$ & $91(45.7)$ & $90(46.9)$ & $1(14.3)$ & 0.09 \\
\hline$<100 \mathrm{mg} / \mathrm{dL}^{\ddagger}$ & $150(75.4)$ & $148(77.1)$ & $2(28.6)$ & $<0.01$ \\
\hline \multicolumn{5}{|l|}{ Distance to target } \\
\hline$<70 \mathrm{mg} / \mathrm{dL}$ & $19.0(9.0-36.0)$ & $19.0(9.0-34.0)$ & $65.0(15.0-77.0)$ & $<0.05$ \\
\hline$<80 \mathrm{mg} / \mathrm{dL}$ & $17.0(7.0-31.0)$ & $16.0(7.0-28.0)$ & $60.5(51.0-67.0)$ & $<0.01$ \\
\hline$<100 \mathrm{mg} / \mathrm{dL}$ & $13.0(6.0-31.0)$ & $11.0(6.0-27.5)$ & $46.0(35.0-47.0)$ & $<0.01$ \\
\hline HDL-C (mg/dL) & $44.0(37.0-53.0)$ & $43.5(37.0-53.0)$ & $45.0(38.0-54.0)$ & 0.86 \\
\hline Non-HDL-C (mg/dL) & $105.0(88.0-124.0)$ & $105.0(87.5-123.0)$ & $147.0(108.0-201.0)$ & $<0.01$ \\
\hline $\mathrm{TC}(\mathrm{mg} / \mathrm{dL})$ & $155.7 \pm 34.7$ & $154.2 \pm 33.4$ & $198.0 \pm 45.5$ & $<0.05$ \\
\hline \multirow[t]{3}{*}{ Triglycerides (mg/dL) } & $106.0(80.0-151.0)$ & $105.5(81.0-151.0)$ & $115.0(77.0-204.0)$ & 0.82 \\
\hline & \multicolumn{4}{|c|}{ Acute coronary syndrome } \\
\hline & $(n=126)$ & $(n=84)$ & $(n=42)$ & \\
\hline LDL-C (mg/dL) & $113.5 \pm 43.9$ & $96.1 \pm 33.8$ & $148.4 \pm 41.0$ & $<0.0001$ \\
\hline$<70 \mathrm{mg} / \mathrm{dL}^{*}$ & $17(13.5)$ & $17(20.2)$ & $0(0)$ & $<0.01$ \\
\hline$<80 \mathrm{mg} / \mathrm{dL}^{\dagger}$ & $30(23.8)$ & $30(35.7)$ & $0(0)$ & $<0.0001$ \\
\hline$<100 \mathrm{mg} / \mathrm{dL}^{\ddagger}$ & $55(43.7)$ & $49(58.3)$ & $6(14.3)$ & $<0.0001$ \\
\hline \multicolumn{5}{|l|}{ Distance to target } \\
\hline$<70 \mathrm{mg} / \mathrm{dL}$ & $45.0(20.0-77.0)$ & $33.0(11.0-50.0)$ & $76.0(49.0-98.0)$ & $<0.0001$ \\
\hline$<80 \mathrm{mg} / \mathrm{dL}$ & $39.5(17.5-73.5)$ & $27.0(13.0-45.0)$ & $66.0(39.0-88.0)$ & $<0.0001$ \\
\hline$<100 \mathrm{mg} / \mathrm{dL}$ & $35.0(15.0-61.0)$ & $18.0(7.0-38.0)$ & $56.0(32.0-80.0)$ & $<0.0001$ \\
\hline HDL-C (mg/dL) & $41.0(35.0-46.0)$ & $41.0(35.0-46.0)$ & $42.0(35.0-46.0)$ & 0.52 \\
\hline Non-HDL-C (mg/dL) & $140.5(105.0-177.0)$ & $116.0(97.0-149.0)$ & $180.0(148.0-214.0)$ & $<0.0001$ \\
\hline $\mathrm{TC}(\mathrm{mg} / \mathrm{dL})$ & $187.4 \pm 49.0$ & $168.2 \pm 38.9$ & $225.9 \pm 44.6$ & $<0.0001$ \\
\hline Triglycerides (mg/dL) & $133.5(100.0-198.0)$ & $127.5(96.5-173.0)$ & $159.0(106.0-241.0)$ & $<0.05$ \\
\hline
\end{tabular}

${ }^{*}$ Goal for very high-risk patients; ${ }^{(14)}$ tgoal defined in Singapore guidelines; ${ }^{(10)} \neq$ previously used goal. ${ }^{(17,18)} \mathrm{HDL}-\mathrm{C}$ : high-density lipoprotein cholesterol; IQR: interquartile range; LDL-C: low-density lipoprotein cholesterol; LLT: lipid-lowering therapy; SD: standard deviation; TC: total cholesterol

Table IV. Predictors of low-density lipoprotein cholesterol $<70 \mathrm{mg} / \mathrm{dL}$ in patients treated with LLT.

\begin{tabular}{|c|c|c|c|c|}
\hline \multirow[t]{2}{*}{ Parameter } & \multicolumn{2}{|c|}{ Stable CHD } & \multicolumn{2}{|c|}{ ACS at admission } \\
\hline & OR $(95 \% \mathrm{CI})$ & p-value & OR $(95 \% \mathrm{CI})$ & p-value \\
\hline Age $\geq 70 y r$ & $0.76(0.35-1.64)$ & 0.48 & $0.65(0.17-2.45)$ & 0.53 \\
\hline Female & $0.84(0.33-2.11)$ & 0.70 & $1.53(0.37-6.43)$ & 0.56 \\
\hline Obesity* & $0.70(0.24-2.01)$ & 0.50 & $0.32(0.05-1.86)$ & 0.20 \\
\hline Waist circumference $^{\dagger}$ & $1.16(0.46-2.98)$ & 0.75 & $0.88(0.21-3.66)$ & 0.86 \\
\hline Current smoking & $1.33(0.48-3.68)$ & 0.58 & $0.32(0.05-2.06)$ & 0.23 \\
\hline Sedentary lifestyle & $1.60(0.71-3.63)$ & 0.26 & $0.34(0.06-2.01)$ & 0.24 \\
\hline Stable angina & $1.54(0.49-4.81)$ & 0.46 & $2.35(0.10-57.21)$ & 0.60 \\
\hline Chronic kidney disease & $0.56(0.11-2.78)$ & 0.48 & $1.60(0.41-6.28)$ & 0.50 \\
\hline Type 2 diabetes mellitus & $2.05(1.01-4.16)$ & 0.05 & $1.68(0.48-5.85)$ & 0.42 \\
\hline History of $\mathrm{CHF}^{\ddagger}$ & $3.34(0.89-12.47)$ & 0.07 & - & - \\
\hline Hypertension & $0.92(0.41-2.03)$ & 0.83 & $0.32(0.05-2.04)$ & 0.23 \\
\hline Statin dose $>20 \mathrm{mg} /$ day $^{\S}$ & $0.99(0.97-1.02)$ & 0.55 & $1.02(0.98-1.06)$ & 0.40 \\
\hline
\end{tabular}

*Body mass index $>30 \mathrm{~kg} / \mathrm{m}^{2}$. †Male $>102 \mathrm{~cm}$, female $>88 \mathrm{~cm}$. ₹Odds ratio for ACS not included in model owing to high number of missing values. $\S A$ torvastatin equivalent. ACS: acute coronary syndrome; CHD: coronary heart disease; CHF: congestive heart failure; Cl: confidence interval; lipid-lowering therapy; OR: odds ratio 

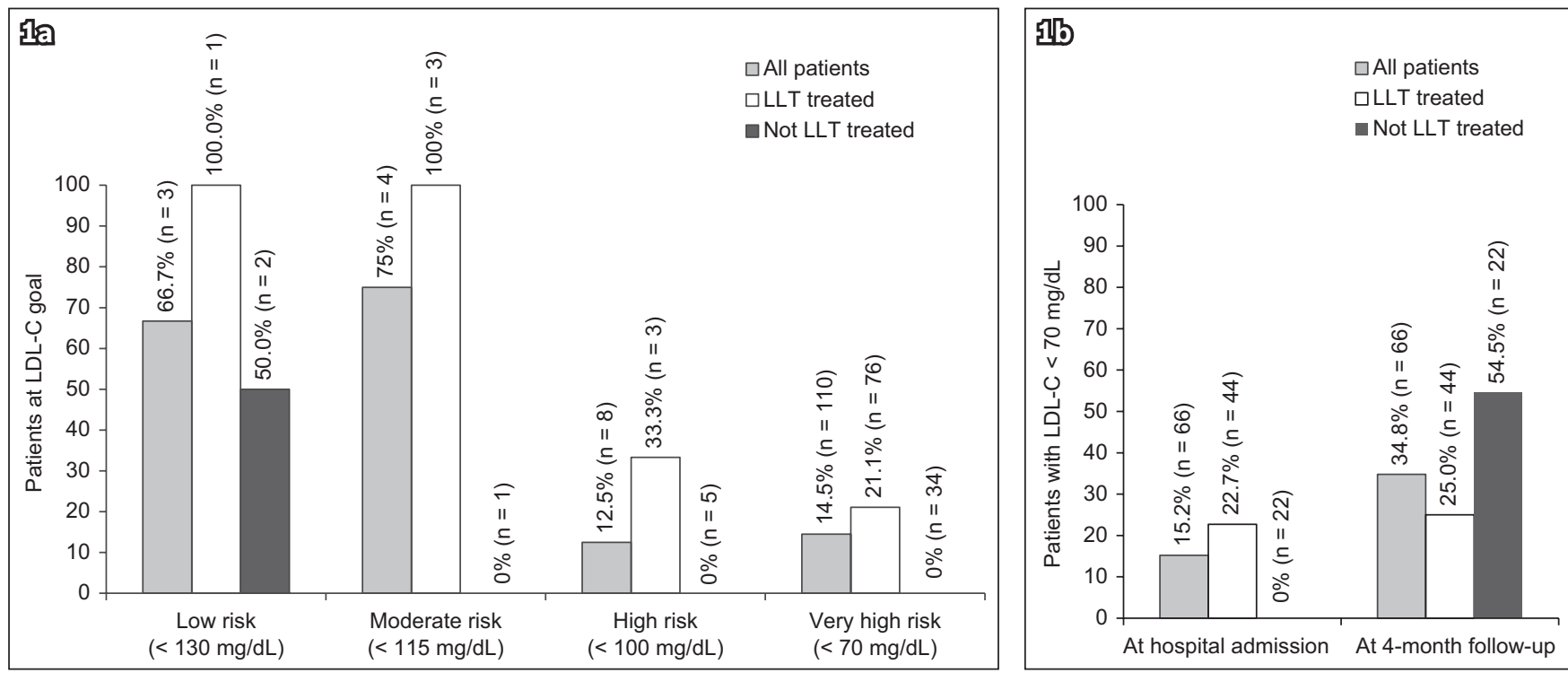

Fig. 1 Bar charts show low-density lipoprotein cholesterol goal achievement (a) at admission by pre-acute coronary syndrome risk level; and (b) at admission and four-month follow-up for patients with available lipid levels at both time points. Risk levels and goals are based on European Society of Cardiology/European Atherosclerosis Society 2011 guidelines. ${ }^{(14)}$ Value of $\mathrm{n}$ is the number of patients in each category. LDL-C: low-density lipoprotein cholesterol; LLT: lipid-lowering therapy

Table V. Use of lipid-lowering therapy (LLT).

\begin{tabular}{|c|c|c|c|}
\hline \multirow[t]{3}{*}{ Parameter } & \multicolumn{3}{|c|}{ No. $(\%) /$ mean \pm SD } \\
\hline & \multirow{2}{*}{$\begin{array}{c}\text { CHD } \\
\text { Baseline }(n=199)\end{array}$} & \multicolumn{2}{|c|}{ ACS } \\
\hline & & Admission $(n=126)$ & 4-month follow-up $(n=113)$ \\
\hline LLT & $192(96.5)$ & $83 / 125(66.4)$ & $106 / 108(98.1)$ \\
\hline Statin therapy & 192/192 (100.0) & 79/83 (95.2) & $105 / 106(99.1)$ \\
\hline Atorvastatin & 67/192 (34.9) & $17 / 79(21.5)$ & $78 / 105(74.3)$ \\
\hline Fluvastatin & $0 / 192(0)$ & $0 / 79(0)$ & $0 / 105(0)$ \\
\hline Lovastatin & $8 / 192(4.2)$ & $5 / 79(6.3)$ & $1 / 105(1.0)$ \\
\hline Pitavastatin & $0 / 192(0)$ & $1 / 79(1.3)$ & $0 / 105(0)$ \\
\hline Pravastatin & $0 / 192(0)$ & $0 / 79(0)$ & $0 / 105(0)$ \\
\hline Rosuvastatin & $29 / 192(15.1)$ & $2 / 79(2.5)$ & $3 / 105(2.9)$ \\
\hline Simvastatin & $88 / 192(45.8)$ & $53 / 79(67.1)$ & $22 / 105(21.0)$ \\
\hline Unknown & $0 / 192(0)$ & $1 / 79(1.3)$ & $1 / 105(1.0)$ \\
\hline Statin daily dose* (mg/day) & $23 \pm 15(n=192)$ & $19 \pm 14(n=78)$ & $34 \pm 18(n=104)$ \\
\hline Statin monotherapy & $159 / 192(82.8)$ & $73 / 83(88.0)$ & $98 / 106(92.5)$ \\
\hline Non-statin monotherapy & $0 / 192(0)$ & $4 / 83(4.8)$ & $1 / 106(0.9)$ \\
\hline Statin + ezetimibe & 18/192 (9.4) & $2 / 83(2.4)$ & $3 / 106(2.8)$ \\
\hline Statin + other non-statin ${ }^{\dagger}$ & 15/192 (7.8) & $4 / 83(4.8)$ & $4 / 106(3.8)$ \\
\hline
\end{tabular}

Value of $\mathrm{n}$ is stated when missing data was excluded from the analysis. *Statin dosage normalised to atorvastatin potency. ${ }^{(16)}+$ Including fibrates, nicotinic acid and omega-3 fatty acids. ACS: acute coronary syndrome; CHD: coronary heart disease; SD: standard deviation

The majority of patients $(96.5 \%)$ in the stable CHD cohort were being treated with some form of LLT at the time of their outpatient visit (Table V). Treatment included a statin in all cases; $82.8 \%$ of patients took it as monotherapy, $9.4 \%$ in combination with ezetimibe and $7.8 \%$ in combination with another non-statin medication. Simvastatin (45.8\%) was the most commonly used statin, followed by atorvastatin (34.9\%) and rosuvastatin (15.1\%). When normalised to atorvastatin potency, the mean daily dosage was $23 \pm 15 \mathrm{mg}$. Treatment did not appear to differ based on patient ethnicity.

In the ACS cohort, $66.4 \%$ of patients were being treated with LLT prior to hospital admission (Table V). A statin was included as part of the therapy in $95.2 \%$ and as monotherapy in $88.0 \%$ of cases, and used in combination with ezetimibe (2.4\%) or other non-statin medication $(4.8 \%)$ in a small number of patients. Simvastatin was also the most commonly prescribed statin $(67.1 \%)$, followed by atorvastatin $(21.5 \%)$ and lovastatin $(6.3 \%)$. The atorvastatin-equivalent daily dosage was $19 \pm 14 \mathrm{mg}$. At the four-month follow-up, $98.1 \%$ of patients were being treated with some form of LLT. In all but one case, a statin was included in the treatment regimen. Use of non-statins remained low: $2.8 \%$ of patients took a statin plus ezetimibe and $3.8 \%$ took a statin with other non-statins. At four months, atorvastatin was the statin of 
choice for $74.3 \%$ of patients, a significant increase from baseline, followed by simvastatin at $21.0 \%$. The atorvastatin-equivalent daily dosage rose to $34 \pm 18 \mathrm{mg}$ at four months. Treatment did not appear to differ based on patient ethnicity.

We calculated that doubling the statin dose for any LLTtreated patients not at goal and not taking atorvastatin $80 \mathrm{mg}$ would slightly increase LDL-C goal achievement (Fig. 2). For the $<70 \mathrm{mg} / \mathrm{dL}$ goal, the proportion of CHD patients could rise to $36.5 \%$, from the current proportion of $28.1 \%$ using the existing treatment. A further $24 \%$ reduction in LDL-C, which may be expected if ezetimibe were to be added to the current LLT regimen, was predicted to greatly increase goal achievement to $71.4 \%$. For the ACS cohort, there was potential for goal achievement to increase from $20.2 \%$ to $25.0 \%$ by doubling the dose of statin for those not at goal and not on atorvastatin $80 \mathrm{mg}$. A further increase to $50.0 \%$ may be expected with the addition of ezetimibe.

The Kaplan-Meier estimates for mortality during follow-up did not vary greatly between the patients treated with LLT prior to hospital admission and those who were not treated (3.9\% vs. $5.6 \% ; p=0.69)$. Rates of non-fatal events were low and KaplanMeier estimates did not differ significantly between groups, with the exception of a requirement for $\mathrm{PCl}$, which was less common in the LLT compared to the no-LLT group ( $5.3 \%$ vs. $17.6 \%$; $p<0.05)$. Overall, rehospitalisation estimates were calculated to be $25.3 \%$ and $29.4 \%$ for the LLT and no-LLT groups, respectively $(p=0.59)$.

\section{DISCUSSION}

The majority of patients included in the Singapore cohort of DYSIS II had an elevated level of LDL-C, with few meeting the ESC guideline's goal for this very-high-risk group. LLT was commonly used, but treatment was not optimised and often inadequate.

Comorbidities and cardiovascular risk factors were prevalent in the stable CHD cohort. This likely contributed to the almost universal utilisation of LLT in this group. As only a small number of $\mathrm{CHD}$ patients were not treated, it was not possible to make accurate comparisons between the characteristics of the treatment and non-treatment groups. The ACS cohort, on the other hand, displayed significant differences depending on whether the patient was being treated with LLT. The LLT group was older and comorbidities were more prevalent, indicating that therapy was often initiated in order to address the patient's poor state of health. For those not on LLT at the time of the ACS, the lower rate of comorbidities may suggest that it was the patients' first contact with a physician for cardiovascular disease.

The mean LDL-C level of the stable CHD cohort was not much higher than the recommended ESC/EAS goal of $<70 \mathrm{mg} / \mathrm{dL}$; however, the proportion of patients achieving this value was low, at just over a quarter. For the LLT-treated group, the distance to goal was only $19.0 \mathrm{mg} / \mathrm{dL}$, indicating that small improvements in the management of hyperlipidaemia could increase the proportion of patients who meet the goal. On the other hand, patients who were not treated with LLT displayed a large distance to goal of $65.0 \mathrm{mg} / \mathrm{dL}$. Even when the less stringent goals of $<80 \mathrm{mg} / \mathrm{dL}$ and $<100 \mathrm{mg} / \mathrm{dL}$ were evaluated, ${ }^{(10,19,20)}$ achievement remained

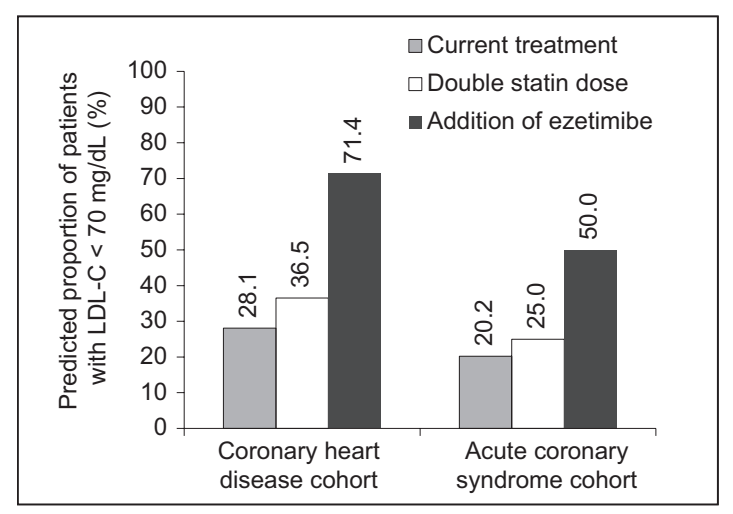

Fig. 2 Bar chart shows hypothetical low-density lipoprotein cholesterol (LDL-C) reductions due to doubling the statin dose, defined according to Weng et al, ${ }^{(16)}$ and adding ezetimibe, defined as $24 \%$ based on the IMPROVE-IT study. ${ }^{(15)}$ Data includes only patients treated with lipid-lowering therapy at baseline.

low. The data suggested that a proportion of patients known to be at very high cardiovascular risk had severe hyperlipidaemia that was poorly managed.

The ACS cohort displayed extremely high LDL-C levels, with the no-LLT group having mean levels that were more than double the ESC/EAS recommended goal for patients at very high cardiovascular risk. Although the treated patients had superior LDL-C values, only $20.2 \%$ attained the goal and there was a high distance to goal. Attainment of the $<80 \mathrm{mg} / \mathrm{dL}$ and $<100 \mathrm{mg} / \mathrm{dL}$ goals was not much better, ${ }^{(10,19,20)}$ with less than half of the overall cohort meeting these goals. Of the patients with follow-up information available, only $61.1 \%$ had a further lipid profile documented within four months after the ACS. The European guidelines that were available at the time of data collection recommend that lipid values are rechecked 4-6 weeks after an ACS, while the National Cholesterol Education ProgramAdult Treatment Panel III report advocates repeat testing six weeks after LLT initiation or dose adjustment. ${ }^{(16,19)}$ The specific Singapore lipid guidelines at the time did not make any specific recommendation, ${ }^{(21)}$ which may explain the lack of retesting; however, the most recent version states that a repeat lipid profile should be obtained three months after a myocardial infarction. ${ }^{(10)}$ Closer adherence to current guidelines may help to improve patient monitoring and, consequently, LDL-C goal achievement in the future.

When follow-up lipid values were considered, there was little change in the proportion of patients treated with LLT prior to hospital admission who met the LDL-C goal (25.0\%). This was despite a significant increase in the mean atorvastatin-equivalent daily statin dosage. The limited change was possibly because patients with higher LDL-C levels were more likely to have a repeat lipid test; however, the small sample size should also be taken into account. On the other hand, a greater proportion of the patients who were not initially treated reached the goal of $<70 \mathrm{mg} / \mathrm{dL}$, likely as a result of initiation of LLT after the ACS event, although goal achievement remained suboptimal. Poor LDL-C goal achievement was previously reported for patients with cardiovascular disease in Singapore. The Achievement in Singapore of Cholesterol Targets (A-SACT) study reported that only 
approximately $30 \%$ of $\mathrm{CHD}$ patients achieved their recommended goal of $<100 \mathrm{mg} / \mathrm{dL}$, with only around $6 \%$ reaching a value of $<70 \mathrm{mg} / \mathrm{dL}$. ${ }^{(22)}$ In a cohort of patients who had suffered an ACS, Chin et al reported an LDL-C level of $<70 \mathrm{mg} / \mathrm{dL}$ for $36.7 \%$ of patients four months after discharge, which decreased to $23.4 \%$ at 12 months. ${ }^{(13)}$ The data collected in DYSIS II supports the observation that hyperlipidaemia appears to be a significant issue in Singapore. With the increasing prevalence of other cardiovascular risk factors, including obesity and Type $2 \mathrm{DM}^{(4)}$ rates of adverse events are likely to increase rapidly if more effective management is not implemented.

A logistic regression model was used to identify factors that may increase the likelihood of an LLT-treated patient meeting the LDL-C goal of $<70 \mathrm{mg} / \mathrm{dL}$. Among them, Type 2 DM was the only factor found to be associated with goal achievement in the $\mathrm{CHD}$ cohort, while none of the variables entered into the ACS model were found to be predictive. The effect of Type 2 DM in the CHD patients can be seen in the distinct lipid profile that is characteristic of the condition, with low HDL-C and high triglyceride levels occurring in combination with less severely elevated LDL-C levels. ${ }^{(23)}$ DM was also predictive for meeting LDL-C goals in the statin-treated global cohort of DYSIS. ${ }^{(11)}$ Furthermore, in the combined European and Canadian cohorts, higher proportions of subjects with DM were reported to have low HDL-C and elevated triglyceride levels compared to those without DM, with a lower proportion not meeting the LDL-C goal. ${ }^{(24)}$

Our examination of the pharmacological management of hyperlipidaemia in CHD and ACS patients in Singapore found widespread use of LLT. In the stable CHD cohort, almost all patients were being treated with LLT. However, the mean atorvastatin-equivalent dosage was quite low and there was little use of combination therapies. In the ACS cohort at baseline, the mean statin dosage for the LLT patients was even lower, with hardly any use of combination regimens. Many previous studies have demonstrated the benefits of LLT for reducing LDL-C levels and cardiovascular events; ${ }^{(25)}$ furthermore, more intensive LLT has been shown to provide superior outcomes. ${ }^{(26,27)}$ However, it appears that the CHD and ACS patients included in the Singapore cohort of DYSIS II were not treated with sufficiently intensified LLT.

The ESC/EAS guidelines recommend that high-dose statin therapy should be initiated within the first 1-4 days after an ACS, ${ }^{(16)}$ while the most recent ACC/AHA cholesterol guidelines advocate high-intensity statin therapy for patients with clinical atherosclerotic cardiovascular disease. ${ }^{(8)}$ Accordingly, by the four-month follow-up, most of the ACS cohort in the present study were being treated with LLT. However, the low mean daily dosage indicated that statin treatment was not maximised in many cases. This is in agreement with the A-SACT study, where patients were found to be more likely to achieve their LDL-C goal if they were being treated with a higher-potency statin, but the majority were taking one with low or very low potency. ${ }^{(22)}$ Similarly, Chin et al reported low statin doses post ACS for patients who did not meet their LDL-C goal. ${ }^{(13)}$ At the time of this study, atorvastatin and rosuvastatin (the more potent statins available), cost more than simvastatin, which was the most commonly used statin in clinical practice in Singapore. There are probably a number of other reasons for this apparent reluctance to prescribe high-intensity statin therapy, such as unfamiliarity with the 2013 ACC/AHA guidelines and with what constitutes a high dosage among physicians in Singapore. ${ }^{(28)}$ There was also a perception that Asian patients would be less tolerant to high doses of statins. ${ }^{(28)} \mathrm{A}$ further potential reason is fear of side effects such as myopathy and elevated liver enzymes, which have been shown to be dose-dependent. ${ }^{(27,29)}$

A further approach to intensification of LLT is the addition of ezetimibe to statin therapy. This was shown to lower LDL-C by approximately $24 \%$ more than with the statin alone $\mathrm{e}^{(17)}$ and provide superior results compared to up-titration of the statin dosage. ${ }^{(30)}$ Use of a statin in combination with ezetimibe was low in the CHD cohort and even more uncommon in the patients admitted to hospital with an ACS. We calculated that the additional LDL-C reduction of about $24 \%$ would have greatly increased goal achievement for patients in both cohorts who were not at goal. ${ }^{(17)}$ Hence, greater use of this drug may be one approach to improving the proportions of patients in Singapore who meet the recommended LDL-C goals.

Few ACS patients died during the relatively short followup period and only small numbers experienced a non-fatal cardiovascular event. It was therefore not possible to identify associations between lipid levels, LLT use and outcomes.

The main limitation of the present study is the small number of patients who had a repeat lipid test after discharge from hospital. This reduced the accuracy of the LDL-C goal achievement values calculated at the four-month follow-up. Similarly, the low number of deaths and adverse events during the four months made it difficult to evaluate the association between lipid levels and cardiovascular health outcomes. It should also be noted that the $\mathrm{CHD}$ cohort may not be representative of all patients with stable $\mathrm{CHD}$, as it is likely that patients who had previously achieved satisfactory lipid levels may have been discharged to primary care. As the DYSIS II data was collected in specialist cardiology clinics, it may only include the sicker patients and those who were struggling to meet LDL-C goals for various reasons. There were also some limitations to the multivariate analysis. In addition to the relatively small sample size, covariates such as medication costs and side effects were not included.

In conclusion, in patients with stable or acute CHD in Singapore, LDL-C levels were greatly in excess of those recommended by experts. Although the different guidelines vary, there is a general consensus that patients at very high cardiovascular risk, such as those in the present study, require intensive LLT and repeat lipid tests. It is therefore clear that significant improvements are required in how these patients are monitored and treated.

\section{ACKNOWLEDGEMENTS}

We would like to thank Katherine Smith (IPPMed, Spain) for her assistance in preparing this manuscript. This study was funded by Merck \& Co Inc, Kenilworth, NJ, USA. 


\section{CONFLICTS OF INTEREST}

Asst Prof Chin Chee Tang received an honorarium from, was on the advisory board, and spoke for AstraZeneca, Medtronic and Terumo. Research support was received from AstraZeneca and Terumo, and education support from Abbott Vascular, Boston Scientific, Biosensors, Biotronik, Orbus-Neich and Terumo. Jee Seong Lim, Weixuan Yu, Dominik Lautsch, Baishali Ambegaonkar and Philippe Brudi are employees of the funder of DYSIS II (Merck \& Co Inc, Kenilworth, NJ, USA).

\section{REFFERENCES}

1. Wong ND. Epidemiological studies of $\mathrm{CHD}$ and the evolution of preventive cardiology. Nat Rev Cardiol 2014; 11:276-89.

2. Ohira T, Iso H. Cardiovascular disease epidemiology in Asia: an overview. Circ J 2013; 77:1646-52.

3. Meng Khoo C, Tai ES. Trends in the incidence and mortality of coronary heart disease in asian pacific region: the Singapore experience. J Atheroscler Thromb 2014; 21 Suppl 1:S2-8.

4. Yan P, Tan EK, Choo JC, et al. Statin-centric versus low-density lipoproteincentric approach for atherosclerotic cardiovascular disease prevention: a Singapore perspective. Singapore Med J 2016; 57:360-7.

5. Zhang X, Patel A, Horibe H, et al; Asia Pacific Cohort Studies Collaboration. Cholesterol, coronary heart disease, and stroke in the Asia Pacific region. Int J Epidemiol 2003; 32:563-72.

6. Baigent C, Keech A, Kearney PM, et al; Cholesterol Treatment Trialists' (CTT) Collaborators. Efficacy and safety of cholesterol-lowering treatment: prospective meta-analysis of data from 90,056 participants in 14 randomised trials of statins. Lancet 2005; 366:1267-78.

7. Catapano AL, Graham I, De Backer G, et al; ESC Scientific Document Group. 2016 ESC/EAS guidelines for the management of dyslipidaemias. Eur Heart J 2016; 37:2999-3058.

8. Stone NJ, Robinson JG, Lichtenstein AH, et al; American College of Cardiology/ American Heart Association Task Force on Practice Guidelines. 2013 ACC/ $\mathrm{AHA}$ guideline on the treatment of blood cholesterol to reduce atherosclerotic cardiovascular risk in adults: a report of the American College of Cardiology/ American Heart Association Task Force on Practice Guidelines. J Am Coll Cardiol 2014; 63(25 Pt B):2889-934.

9. Teramoto T, Sasaki J, Ishibashi S, et al; Japan Atherosclerosis Society. Executive summary of the Japan Atherosclerosis Society (JAS) guidelines for the diagnosis and prevention of atherosclerotic cardiovascular diseases in Japan-2012 version. J Atheroscler Thromb 2013; 20:517-23.

10. Tai ES, Chia BL, Bastian AC, et al. Ministry of Health Clinical Practice Guidelines: Lipids. Singapore Med J 2017; 58:155-66.

11. Gitt AK, Lautsch D, Ferrieres J, et al. Low-density lipoprotein cholesterol in a global cohort of 57,885 statin-treated patients. Atherosclerosis 2016; 255:200-9.

12. Park JE, Chiang CE, Munawar M, et al. Lipid-lowering treatment in hypercholesterolaemic patients: the CEPHEUS Pan-Asian survey. Eur J Prev Cardiol 2012; 19:781-94.

13. Chin CW, Gao F, Le T, Tan R. Lipid goal attainment and prescription behavior in asian patients with acute coronary syndromes: experience from a tertiary hospital. Clin Med Insights Cardiol 2013; 7:51-7.

14. Gitt AK, Lautsch D, Ferrières J, et al. Cholesterol target value attainment and lipid-lowering therapy in patients with stable or acute coronary heart disease:
Results from the Dyslipidemia International Study II. Atherosclerosis 2017; 266:158-166.

15. Poh KK, Ambegaonkar B, Baxter CA, et al. Low-density lipoprotein cholesterol target attainment in patients with stable or acute coronary heart disease in the Asia-Pacific region: results from the Dyslipidemia International Study II. Eur J Prev Cardiol 2018; 25:1950-63.

16. European Association for Cardiovascular Prevention \& Rehabilitation, Reiner Z, Catapano AL, et al; ESC Committee for Practice Guidelines (CPG) 20082010 and 2010-2012 Committees. ESC/EAS Guidelines for the management of dyslipidaemias: the Task Force for the management of dyslipidaemias of the European Society of Cardiology (ESC) and the European Atherosclerosis Society (EAS). Eur Heart J 2011; 32:1769-818.

17. Cannon CP, Blazing MA, Giugliano RP, et al; IMPROVE-IT Investigators. Ezetimibe added to statin therapy after acute coronary syndromes. N Engl J Med 2015; 372:2387-97.

18. Weng TC, Yang YH, Lin SJ, Tai SH. A systematic review and meta-analysis on the therapeutic equivalence of statins. J Clin Pharm Ther 2010; 35:139-51.

19. National Cholesterol Education Program (NCEP) Expert Panel on Detection, Evaluation, and Treatment of High Blood Cholesterol in Adults (Adult Treatment Panel III). Third Report of the National Cholesterol Education Program (NCEP) Expert Panel on Detection, Evaluation, and Treatment of High Blood Cholesterol in Adults (Adult Treatment Panel III) final report. Circulation 2002; 106:3143-421.

20. Graham I, Atar D, Borch-Johnsen K, et al. European guidelines on cardiovascular disease prevention in clinical practice: full text. Fourth Joint Task Force of the European Society of Cardiology and other societies on cardiovascular disease prevention in clinical practice (constituted by representatives of nine societies and by invited experts). Eur J Cardiovasc Prev Rehabil 2007; 14 Suppl 2:S1-113.

21. Ministry of Health, Singapore. Clinical Practice Guidelines: Lipids. 2006. Available at: https://www.moh.gov.sg/content/dam/moh_web/HPP/Doctors/ cpg_medical/withdrawn/cpg_Lipids-May\%202006.pdf. Accessed February 2018.

22. Ho KT, Chin KW, Ng KS, et al. The A-SACT (Achievement in Singapore of Cholesterol Targets) study in patients with coronary heart disease. Am J Cardiovasc Drugs 2006; 6:383-91.

23. Taskinen MR, Borén J. New insights into the pathophysiology of dyslipidemia in type 2 diabetes. Atherosclerosis 2015; 239:483-95.

24. Leiter LA, Lundman P, da Silva PM, et al; DYSIS Investigators. Persistent lipid abnormalities in statin-treated patients with diabetes mellitus in Europe and Canada: results of the Dyslipidaemia International Study. Diabet Med 2011; 28:1343-51.

25. Cholesterol Treatment Trialists' (CTT) Collaborators, Kearney PM, Blackwell L, et al. Efficacy of cholesterol-lowering therapy in 18,686 people with diabetes in 14 randomised trials of statins: a meta-analysis. Lancet 2008; 371:117-25.

26. Cannon CP, Steinberg BA, Murphy SA, Mega JL, Braunwald E. Meta-analysis of cardiovascular outcomes trials comparing intensive versus moderate statin therapy. J Am Coll Cardiol 2006; 48:438-45.

27. Josan K, Majumdar SR, McAlister FA. The efficacy and safety of intensive statin therapy: a meta-analysis of randomized trials. CMAJ 2008; 178:576-84.

28. Setia S, Fung SS, Waters DD. Doctors' knowledge, attitudes, and compliance with 2013 ACC/AHA guidelines for prevention of atherosclerotic cardiovascular disease in Singapore. Vasc Health Risk Manag 2015; 11:303-10.

29. Morrissey RP, Diamond GA, Kaul S. Statins in acute coronary syndromes: do the guideline recommendations match the evidence? J Am Coll Cardiol 2009; 54:1425-33.

30. Foody JM, Toth PP, Tomassini JE, et al. Changes in LDL-C levels and goal attainment associated with addition of ezetimibe to simvastatin, atorvastatin, or rosuvastatin compared with titrating statin monotherapy. Vasc Health Risk Manag 2013; 9:719-27. 